\title{
Cannabis use and resting state functional connectivity in adolescent bipolar disorder
}

\author{
Alysha A. Sultan, PhD; Megan A. Hird, MSc; Mikaela K. Dimick, BA; \\ Bradley J. MacIntosh, PhD; Benjamin I. Goldstein, MD, PhD
}

\begin{abstract}
Background: Adolescents with bipolar disorder have high rates of cannabis use, and cannabis use is associated with increased symptom severity and treatment resistance in bipolar disorder. Studies have identified anomalous resting-state functional connectivity among reward networks in bipolar disorder and cannabis use independently, but have yet to examine their convergence. Methods: Participants included 134 adolescents, aged 13 to 20 years: 40 with bipolar disorder and lifetime cannabis use, 31 with bipolar disorder and no history of cannabis use, and 63 healthy controls without lifetime cannabis use. We used a seed-to-voxel analysis to assess the restingstate functional connectivity of the amygdala, the nucleus accumbens and the orbitofrontal cortex, regions implicated in bipolar disorder and cannabis use. We used a generalized linear model to explore bivariate correlations for each seed, controlling for age and sex. Results: We found 3 significant clusters. Resting-state functional connectivity between the left nucleus accumbens seed and the left superior parietal lobe was negative in adolescents with bipolar disorder and no history of cannabis use, and positive in healthy controls. Resting-state functional connectivity between the right orbitofrontal cortex seed and the right lateral occipital cortex was positive in adolescents with bipolar disorder and lifetime cannabis use, and negative in healthy controls and adolescents with bipolar disorder and no history of cannabis use. Resting-state functional connectivity between the right orbitofrontal cortex seed and right occipital pole was positive in adolescents with bipolar disorder and lifetime cannabis use, and negative in adolescents with bipolar disorder and no history of cannabis use. Limitations: The study did not include a cannabis-using control group. Conclusion: This study provides preliminary evidence of cannabis-related differences in functional reward circuits in adolescents with bipolar disorder. Further studies are necessary to evaluate whether the present findings reflect consequences of or predisposition to cannabis use.
\end{abstract}

\section{Introduction}

Bipolar disorder affects approximately $2 \%$ to $5 \%$ of adolescents worldwide, and is characterized by recurrent mood episodes, including depression and mania or hypomania. ${ }^{1,2}$ Bipolar disorder is associated with increased rates of substance use and substance use disorders., ${ }^{3,4}$ Approximately 1 in 3 adolescents with bipolar disorder have comorbid substance use disorders, and cannabis is the most commonly used drug., ${ }^{5,6}$ Cannabis use and cannabis use disorder have been associated with a more severe course of bipolar disorder, including delayed recovery, increased number of symptoms and episodes, increased functional disability and decreased treatment adherence and response.., $7-9$

Previous structural and functional imaging studies have implicated reward-related brain circuits in cannabis use and bipolar disorder independently. Regions identified in these studies are relevant to reward-related decisions and behaviours such as impulse control, decision-making and emotional regulation. ${ }^{10-13}$ Key hubs in reward circuits include the prefrontal cortex, the amygdala, the nucleus accumbens and the striatum. ${ }^{14}$ However, despite the relevance of reward circuits to both cannabis use and bipolar disorder, few studies have examined these topics together, ${ }^{15-17}$ and none have examined the resting-state functional connectivity correlates of cannabis use in bipolar disorder.

Adolescence is a period of dynamic neurobiological and behavioural change. ${ }^{18}$ This critical developmental epoch is also a period of heightened vulnerability to the use and deleterious effects of exogenous substances on the brain. ${ }^{19,20}$ Cannabis comprises more than 100 components known as cannabinoids, of which tetrahydrocannabinol (the main psychoactive component of cannabis) and cannabidiol are among the most studied. ${ }^{21}$ Both compounds interact with the endocannabinoid system, an intracellular signalling system found in the brain and throughout the body.22 There are 2 primary cannabinoid receptors: type 1 is most concentrated in the brain, and type 2 is most concentrated in the periphery. ${ }^{21}$ The endocannabinoid

Correspondence to: Benjamin I. Goldstein, Centre for Addiction and Mental Health, 80 Workman Way, Toronto, ON M6J 1H4; benjamin.goldstein@camh.ca

Submitted Dec. 10, 2020; Revised Apr. 21, 2021; Accepted Jul. 4, 2021

Cite as: J Psychiatry Neurosci 2021 October 8;46(5). doi: 10.1503.jpn/200228 
system is one of the most important physiologic systems involved in establishing and maintaining human health, with complex actions in the nervous system, the immune system and multiple organ systems. ${ }^{23,24}$

Resting-state functional connectivity represents a specific functional MRI technique that measures the temporal synchronization of functional MRI signals between spatially distinct brain areas. ${ }^{25-28}$ To our knowledge, 1 task-based functional MRI study in adolescent bipolar disorder has used a cannabis-cue paradigm to elicit brain activation patterns during visual stimuli (i.e., neutral v. cannabis-related images). ${ }^{17}$ Adolescents with bipolar disorder but no cannabis use disorder $(n=14)$ exhibited greater activation than adolescents with bipolar disorder and comorbid cannabis use disorder $(n=25)$ or controls $(n=15)$ in the amygdala, the nucleus accumbens, the thalamus and the striatum - regions involved in emotional processing and reward. There were no significant differences for adolescents with bipolar disorder and cannabis use disorder compared to controls. ${ }^{17}$

The purpose of the present study was to investigate differences in resting-state functional connectivity in the reward network among the following groups: adolescents with bipolar disorder and lifetime cannabis use (from infrequent cannabis use to cannabis use disorder); adolescents with bipolar disorder and no history of cannabis use; and healthy controls. Using a seed-to-voxel analysis, we examined resting-state functional connectivity in 3 different regions of interest (ROIs): the amygdala, the nucleus accumbens and the orbitofrontal cortex. We hypothesized that adolescents with bipolar disorder and lifetime cannabis use would have different resting-state functional connectivity patterns in the chosen ROIs compared to adolescents with bipolar disorder and no history of cannabis use, and to healthy controls. Because of a paucity of previous related research, these hypotheses did not specify directionality (i.e., increased v. decreased). Identifying the neural signatures of cannabis use in adolescents with bipolar disorder can offer important insights into the functional disruptions that may underlie the predisposition to and consequences of cannabis use in this population, in whom the clinical correlates of such use is particularly pernicious.

\section{Methods}

This study was approved by the research ethics board at Sunnybrook Health Sciences Centre. Written informed consent was obtained from all participants and their parents or guardians.

\section{Participants}

The analyzed sample included 134 English-speaking adolescents aged 13 to 20 years: adolescents with bipolar disorder and lifetime cannabis use $(n=40)$; adolescents with bipolar disorder and no history of cannabis use $(n=31)$; and healthy controls $(n=63)$. Participants with bipolar disorder were recruited from the Centre for Youth Bipolar Disorder, a subspecialty clinical research program at Sunnybrook Health
Sciences Centre in Toronto, Ontario, Canada. Healthy controls were recruited primarily via community advertisements.

We assessed current and lifetime psychiatric diagnoses for all participants using the Kiddie Schedule for Affective Disorders and Schizophrenia for School-Age Children, Present and Lifetime Version (KSADS-PL), ${ }^{29}$ a semistructured interview completed with both the adolescents and their parent(s) or guardian(s). We used the expanded KSADS Mania Rating Scale $^{30}$ and the KSADS Depression Rating Scale ${ }^{31}$ to assess mood symptoms. We also used the KSADS-PL interview to assess cannabis use. Participants were asked whether they had ever used cannabis before, and for those who answered yes, we administered the KSADS substance use supplement to determine if the participant met the diagnostic criteria for cannabis use disorder. Participants were classified as adolescents with bipolar disorder and lifetime cannabis use if they reported any lifetime cannabis use. We obtained participants' lifetime history of sexual and physical abuse using the KSADS-PL posttraumatic stress disorder screening questions. We measured current and lifetime functioning of each adolescent using the Children's Global Assessment Scale, ${ }^{32}$ and we assessed the socioeconomic status of the parents or guardians using the Hollingshead Four-Factor Index. ${ }^{33}$ All interviewers had bachelor's or master's degrees and had completed rigorous training under the supervision of the principal investigator (B.G.). Diagnoses and symptom ratings were reviewed and confirmed by a licensed child-adolescent psychiatrist. Diagnoses of bipolar disorder I and bipolar disorder II were based on DSM-IV criteria, because our sample was recruited from 2012 to 2019, and the DSM-5 version of the KSADS-PL was not available until December 2016. For bipolar disorder not otherwise specified, we adopted the operationalized criteria specified in the Course and Outcome of Bipolar Illness study: elated mood plus 2 associated mania symptoms (3 if irritable mood only); change of functioning; minimum 4 hours' duration in 24 hours; and 4 or more cumulative lifetime days meeting the criteria. ${ }^{34}$

Exclusion criteria were as follows: unable to provide informed consent; a pre-existing cardiac, autoimmune or inflammatory illness; currently taking any anti-inflammatory, antiplatelet, antilipidemic, antihypertensive or antihyperglycemic agent(s); any infectious illness within the previous 14 days; any MRI contraindications (e.g., any metal in the body, claustrophobia); any severe neurologic or cognitive impairments; and substance dependence in the previous 3 months. Current cannabis abuse, current recreational use and previous cannabis dependence were not relevant exclusion criteria for this study. In addition to the criteria above, healthy controls were also excluded if they had a major psychiatric disorder (i.e., no lifetime mood or psychotic disorder and no anxiety disorder in the previous 3 months), or any first- or second-degree relative with bipolar disorder or a psychotic disorder. The present study was a secondary analysis based on participants from 2 other studies (see funding sources); although cannabis use was not an exclusion criterion for those studies, 9 healthy controls were excluded from the present analyses because of small cell size and the absence of cannabis use disorder in the healthy controls group. 


\section{MRI acquisition}

We collected neuroimaging data using a 3 T Philips Achieva scanner with an 8-channel head receiver coil and body coil transmission. We acquired structural images using $T_{1}$ weighted high-resolution fast-field echo images with the following parameters: repetition time $9.5 \mathrm{~ms}$, echo time $2.3 \mathrm{~ms}$, inversion time $1400 \mathrm{~ms}$, spatial resolution $0.94 \times 1.17 \times$ $1.2 \mathrm{~mm}$, matrix $256 \times 164 \times 140$, flip angle $8^{\circ}$, field of view 240 $\times 191 \mathrm{~mm}^{2}$, scan duration $8 \mathrm{~m} 56 \mathrm{~s}, 140$ slices. We collected resting-state functional MRIs in the axial plane using $T_{2}{ }^{*}$ weighted echo planar imaging with the following parameters: repetition time $1500 \mathrm{~ms}$, echo time $30 \mathrm{~ms}$, spatial resolution $3 \times 3 \times 4 \mathrm{~mm}$, matrix $76 \times 60 \times 28$, flip angle $70^{\circ}$, field of view $230 \times 181 \mathrm{~mm}$, scan duration $5 \mathrm{~m} 50 \mathrm{~s}, 230$ volumes.

During the resting-state scan, participants were instructed to keep their eyes open, focus on a blank screen and think about nothing in particular.

\section{Functional MRI analysis}

We completed preprocessing steps and the following analyses using the CONN toolbox (version 17) in Matlab. ${ }^{35}$ We removed the first 3 volumes of functional data to account for signal equilibration. We completed preprocessing of functional volumes using the default pipeline for volume-based analysis in the CONN toolbox, including functional realignment and unwarping (participant motion estimation and correction), structural and functional translation, slice-timing correction, functional outlier detection (Artifact Detection Tools based identification of outlier scans for scrubbing), structural and functional segmentation, and normalization to Montreal Neurological Institute space (simultaneously grey/white/cerebrospinal fluid segmentation), as well as functional smoothing $(8 \mathrm{~mm}$ full width at half maximum Gaussian filter). Head motion was accounted for in the CONN toolbox by identifying problematic time points using Artifact Detection Tools. Images were considered outliers if the global mean image intensity was more than 3 standard deviations from the mean image intensity for the entire resting scan, or if there was a displacement of more than $1.0 \mathrm{~mm}$ from the previous frame in an $x, y$, or $z$ direction. We also examined all volumes manually for motion outliers (> $2 \mathrm{~mm}$ or $2^{\circ}$ rotation in any direction: $\left.x, y, z\right)$, and excluded participants if they had any volumes with motion outliers (35 participants excluded: 9 adolescents with bipolar disorder and lifetime cannabis use, 7 adolescents with bipolar disorder and no history of cannabis use, and 19 healthy controls). We used CONN's default pipeline for denoising, which combines 2 steps: a linear regression of potential confounds in the blood-oxygen-level-dependent signal (including white matter, cerebrospinal fluid, realignment, scrubbing and the effect of rest), and band-pass filtering (temporal frequencies below $0.008 \mathrm{~Hz}$ or above $0.09 \mathrm{~Hz}$ were removed). After denoising, 2 independent raters examined the histograms from the functional connectivity values, which revealed normally distributed data for all participants who were not excluded for head motion.
We determined seed selection a priori and included the amygdala, nucleus accumbens and orbitofrontal cortex, defined using the FMRIB Software Library Harvard-Oxford structural atlas generated by the CONN toolbox and parcellated into left and right hemispheres.

\section{Statistical analysis}

We analyzed categorical variables using $\chi^{2}$ tests and continuous variables using independent-samples $t$ tests or analysis of variance (for 3-way analyses). We used nonparametric tests (Mann-Whitney $U$ tests, Kruskal-Wallis tests) for variables that were not normally distributed. Two-tailed statistical significance was set at $p<0.05$.

For the functional connectivity analyses, we used a seed-tovoxel approach. We computed bivariate correlation coefficients between the time series for each bilateral seed region and all other voxel blood-oxygen-level-dependent time series to produce seed-to-voxel functional connectivity maps. Connectivity maps were normalized using Fisher $z$ transformation, and $\beta$ values represent Fisher-transformed correlation coefficient values. We used a generalized linear model to investigate 3-way between-group differences, including demeaned age and sex as covariates. ${ }^{35}$ Using $p$ values corrected for false discovery rate, we set the voxel-wise height threshold at $p<0.001$ and cluster thresholding at $p<0.05$. We applied Bonferroni correction for multiple comparisons for the number of between-group contrasts (i.e., $p<0.017$ ). Significant clusters from the generalized linear model analyses were exported as masks to conduct post hoc pair-wise comparisons in an ROI-to-ROI analysis, applying Bonferroni correction for the number of post hoc pair-wise tests (i.e., $p<0.017$ ).

\section{Results}

\section{Demographic and clinical characteristics}

Demographic characteristics for all participants are presented in Table 1. The groups did not differ significantly in terms of age or sex. No healthy controls had a history of cannabis use. Clinical characteristics for adolescents with bipolar disorder are presented in Table 2. Adolescents with bipolar disorder and lifetime cannabis use had more suicide attempts, police contact or arrest, substance use disorders and alcohol dependence than adolescents with bipolar disorder and no history of cannabis use. Among the adolescents with bipolar disorder and lifetime cannabis use, 13 met the criteria for lifetime cannabis use disorder (cannabis abuse $n=13$; cannabis dependence $n=10$ ).

\section{Seed-to-voxel analyses}

Our analysis of adolescents with bipolar disorder and lifetime cannabis use compared to healthy controls and adolescents with bipolar disorder and no history of cannabis use revealed altered between-group resting-state functional connectivity in the left nucleus accumbens and the right orbitofrontal cortex seeds (Table 3). 
Table 1: Demographic characteristics

\begin{tabular}{|c|c|c|c|c|c|c|}
\hline Characteristic & $\begin{array}{l}\text { Bipolar disorder and } \\
\text { lifetime cannabis use } \\
\qquad n=40\end{array}$ & $\begin{array}{l}\text { Bipolar disorder and no } \\
\text { history of cannabis use } \\
\qquad n=31\end{array}$ & $\begin{array}{l}\text { Healthy } \\
\text { controls } \\
n=63\end{array}$ & Test statistic & $p$ value & Effect size \\
\hline Age, yr & $17.49 \pm 1.25$ & $17.39 \pm 1.91$ & $16.90 \pm 1.63$ & $F=2.03$ & 0.14 & $\eta^{2}=0.03$ \\
\hline Female & $25(62.5)$ & $19(61.3)$ & $34(54.0)$ & $\chi^{2}=0.89$ & 0.64 & $V=0.08$ \\
\hline Socioeconomic status ${ }^{*}$ & $4.20 \pm 0.97$ & $4.35 \pm 0.80$ & $4.37 \pm 0.87$ & $H=0.76$ & 0.68 & $\eta^{2}=0.01$ \\
\hline Race or ethnicity, White & $31(77.5)$ & $23(74.2)$ & $32(50.8)$ & $\chi^{2}=9.35$ & $0.009 \S \rrbracket$ & $V=0.26$ \\
\hline Intact family & $22(55.0)$ & $21(67.7)$ & $42(66.7)$ & $\chi^{2}=1.76$ & 0.42 & $V=0.12$ \\
\hline Tanner stage $\dagger$ & $4.48 \pm 0.64$ & $4.39 \pm 0.67$ & $4.29 \pm 0.61$ & $H=2.77$ & 0.25 & $\eta^{2}=0.02$ \\
\hline Body mass index (adjusted), $\mathrm{kg} / \mathrm{m}^{2}$ & $24.46 \pm 4.41$ & $23.57 \pm 4.77$ & $21.91 \pm 3.69$ & $H=11.11$ & $0.004 \S$ & $\eta^{2}=0.08$ \\
\hline \multicolumn{7}{|c|}{ Children's Global Assessment Scale score } \\
\hline Most severe previous episode & $43.30 \pm 8.95$ & $44.73 \pm 8.91$ & - & $U=643.50$ & 0.60 & $d=0.16$ \\
\hline Highest score in past yearł & $67.33 \pm 12.34$ & $69.90 \pm 10.68$ & $89.48 \pm 5.07$ & $F=93.47$ & $<0.001 \S \rrbracket$ & $\eta^{2}=0.58$ \\
\hline Score in past month $\ddagger$ & $64.18 \pm 11.81$ & $65.90 \pm 11.28$ & $89.49 \pm 4.53$ & $F=132.93$ & $<0.001 \S \rrbracket$ & $\eta^{2}=0.66$ \\
\hline \multicolumn{7}{|c|}{$\begin{array}{l}\text { Values are reported as mean } \pm \text { standard error or } n(\%) \text {. } \\
\text { *Based on the Hollingshead Four Factor Index. }{ }^{33} \\
\text { †Measured on a scale of } 1 \text { to } 5 \text {. } \\
\text { †Homogeneity of variance violated; Welsh test reported. } \\
\S \text { Significant at } p<0.05 \text { (adolescents with bipolar disorder and lifetime cannabis use v. healthy controls). } \\
\text { ๆSignificant at } p<0.05 \text { (adolescents with bipolar disorder and no history of cannabis use v. healthy controls). }\end{array}$} \\
\hline
\end{tabular}

The left nucleus accumbens seed yielded a significant difference in resting-state functional connectivity in the left superior parietal lobe $\left(\beta=0.25, F_{2,129}=18.31, p<0.001\right)$, and this finding remained significant after correction for multiple comparisons (Figure 1). Analyses of the left nucleus accumbens to the left superior parietal lobe showed a significant anticorrelation: adolescents with bipolar disorder and no history of cannabis use had negative connectivity, and healthy controls had positive connectivity $\left(\beta=0.31, F_{1,90}=\right.$ $39.34, p<0.001)$. We found no significant differences for adolescents with bipolar disorder and lifetime cannabis use in this analysis.

The right orbitofrontal cortex seed revealed altered restingstate functional connectivity in 3 significant clusters. First, we found a significant difference in resting-state functional connectivity between the right orbitofrontal cortex and the right lateral occipital cortex $\left(\beta=0.18, F_{2,129}=10.93, p<0.001\right)$. Pairwise post hoc analysis of the right orbitofrontal cortex to the right lateral occipital cortex showed a significant anticorrelation: adolescents with bipolar disorder and lifetime cannabis use had positive connectivity, and adolescents with bipolar disorder and no history of cannabis use had negative connectivity $\left(\beta=0.26, F_{1,67}=14.80, p=0.003\right)$. We also found a significant anticorrelation for adolescents with bipolar disorder and lifetime cannabis use and healthy controls: adolescents with bipolar disorder and lifetime cannabis use had positive connectivity, and healthy controls had negative connectivity $(\beta=$ $\left.0.25, F_{1,99}=17.25, p=0.002\right)$. For the right orbitofrontal cortex to the right lateral occipital cortex, we found no significant differences in resting-state functional connectivity between adolescents with bipolar disorder and no history of cannabis use and healthy controls. Second, we found a significant difference in resting-state functional connectivity between the right orbitofrontal cortex and the right occipital pole $(\beta=$ $\left.0.17, F_{2,129}=9.19, p=0.007\right)$. Pair-wise post hoc analysis showed a significant anticorrelation: adolescents with bipolar disorder and lifetime cannabis use had positive connectivity, and adolescents with bipolar disorder and no history of cannabis use had negative connectivity $\left(\beta=0.23, F_{1,67}=15.79, p=\right.$ 0.003). We found no significant differences between healthy controls and either group of adolescents with bipolar disorder for the right orbitofrontal cortex to the right occipital pole. The clusters in the right lateral occipital cortex and right occipital pole survived correction for multiple comparisons (Figure 2). Third, we found a significant difference in restingstate functional connectivity between the right orbitofrontal cortex and the left occipital pole $\left(\beta=0.12, F_{2,129}=7.23, p=\right.$ $0.045)$, but this cluster did not survive correction for multiple comparisons.

We found no significant differences in resting-state functional connectivity originating from the right nucleus accumbens seed, the left orbitofrontal cortex seed or the bilateral amygdala seeds.

We conducted sensitivity analyses using the same model as the original analyses, controlling for alcohol dependence, police contact or arrest and suicide attempts independently. In each case, the findings for the left nucleus accumbens remained significant, but findings for the right orbitofrontal cortex were no longer significant.

\section{Discussion}

The present study used a seed-to-voxel analysis to identify patterns of differential resting-state functional connectivity across adolescents with bipolar disorder and lifetime cannabis use, adolescents with bipolar disorder and no history of cannabis use, and healthy controls. We observed betweengroup differences in 3 significant clusters across 2 seeds in the reward network: the nucleus accumbens and the orbitofrontal cortex. For the left nucleus accumbens seed, we found 
Table 2: Clinical characteristics of bipolar disorder groups

\begin{tabular}{|c|c|c|c|c|c|}
\hline Characteristic & $\begin{array}{l}\text { Bipolar disorder and } \\
\text { lifetime cannabis use } \\
\qquad n=40\end{array}$ & $\begin{array}{l}\text { Bipolar disorder and no } \\
\text { history of cannabis use } \\
\qquad n=31\end{array}$ & Test statistic & $p$ value & Effect size \\
\hline \multicolumn{6}{|l|}{ Bipolar disorder characteristics } \\
\hline Bipolar disorder I & $16(40.0)$ & $8(25.8)$ & $\chi^{2}=2.12$ & 0.35 & $V=0.17$ \\
\hline Bipolar disorder II & $13(32.5)$ & $10(32.3)$ & & & \\
\hline Bipolar disorder NOS & $11(27.5)$ & $13(41.9)$ & & & \\
\hline Age at onset & $15.22 \pm 2.53$ & $14.32 \pm 2.91$ & $U=468.0$ & 0.12 & $d=0.33$ \\
\hline \multicolumn{6}{|l|}{ Clinical characteristics } \\
\hline Lifetime psychosis & $3(7.5)$ & $6(19.4)$ & $\chi^{2}=2.22$ & 0.14 & $V=0.18$ \\
\hline Lifetime suicide attempt(s) & $10(25.0)$ & $1(3.2)$ & $\chi^{2}=6.32$ & $0.01 \dagger$ & $V=0.30$ \\
\hline Lifetime self-injurious behaviour & $19(47.5)$ & $16(51.6)$ & $\chi^{2}=0.19$ & 0.73 & $V=0.04$ \\
\hline Lifetime suicidal ideation & $23(57.5)$ & $19(61.3)$ & $\chi^{2}=0.10$ & 0.75 & $V=0.04$ \\
\hline Police contact or arrest & $12(30.0)$ & $3(9.7)$ & $\chi^{2}=4.33$ & $0.04 \dagger$ & $V=0.25$ \\
\hline Lifetime physical or sexual abuse & $2(5.0)$ & $2(6.5)$ & $\chi^{2}=0.07$ & 0.79 & $V=0.03$ \\
\hline Lifetime psychiatric hospitalization & $21(52.5)$ & $11(35.5)$ & $\chi^{2}=2.04$ & 0.15 & $V=0.17$ \\
\hline Current depression score ${ }^{\star}$ & $15.83 \pm 11.91$ & $15.42 \pm 9.58$ & $U=597.0$ & 0.79 & $d=0.04$ \\
\hline Lifetime depression score ${ }^{*}$ & $30.40 \pm 12.27$ & $28.42 \pm 11.39$ & $U=569.0$ & 0.55 & $d=0.17$ \\
\hline Current mania score* & $9.45 \pm 10.73$ & $8.65 \pm 8.25$ & $U=629.0$ & 0.92 & $d=0.08$ \\
\hline Lifetime mania score ${ }^{*}$ & $30.25 \pm 10.91$ & $30.32 \pm 9.48$ & $t=-0.03$ & 0.98 & $d=0.01$ \\
\hline \multicolumn{6}{|l|}{ Lifetime comorbid diagnoses } \\
\hline Attention-deficit/hyperactivity disorder & $20(50.0)$ & $15(48.4)$ & $\chi^{2}=0.02$ & 0.89 & $V=0.02$ \\
\hline Any anxiety & $30(75.0)$ & $24(77.4)$ & $\chi^{2}=0.06$ & 0.81 & $V=0.03$ \\
\hline Substance use disorder & $14(35.0)$ & $2(6.5)$ & $\chi^{2}=8.15$ & $0.004 \dagger$ & $V=0.34$ \\
\hline Oppositional defiant disorder & $13(32.5)$ & $7(22.6)$ & $\chi^{2}=0.85$ & 0.36 & $V=0.11$ \\
\hline Conduct disorder & $3(7.5)$ & 0 & $\chi^{2}=2.43$ & 0.12 & $V=0.19$ \\
\hline Nicotine use & $9(22.5)$ & $2(6.5)$ & $\chi^{2}=3.44$ & 0.06 & $V=0.22$ \\
\hline Alcohol abuse & $4(10.0)$ & $1(3.2)$ & $\chi^{2}=1.22$ & 0.27 & $V=0.13$ \\
\hline Alcohol dependence & $5(12.5)$ & 0 & $\chi^{2}=4.17$ & $0.04 \dagger$ & $V=0.24$ \\
\hline \multicolumn{6}{|l|}{ Family psychiatric history } \\
\hline Mania/hypomania & $6(15)$ & $8(25.8)$ & $\chi^{2}=1.46$ & 0.23 & $V=0.14$ \\
\hline Depression & $23(57.5)$ & $17(54.8)$ & $\chi^{2}=0.01$ & 0.94 & $V=0.01$ \\
\hline Anxiety & $22(55.0)$ & $14(45.2)$ & $\chi^{2}=0.48$ & 0.49 & $V=0.08$ \\
\hline Attention-deficit/hyperactivity disorder & $11(27.5)$ & $7(22.6)$ & $\chi^{2}=0.16$ & 0.69 & $V=0.05$ \\
\hline \multicolumn{6}{|l|}{ Lifetime medications } \\
\hline Second-generation antipsychotic & $29(72.5)$ & $24(77.4)$ & $\chi^{2}=0.22$ & 0.64 & $V=0.06$ \\
\hline Lithium & $9(22.5)$ & $7(22.6)$ & $\chi^{2}=0.00$ & 0.99 & $V=0.001$ \\
\hline SSRI antidepressant & $14(35.0)$ & $9(29.0)$ & $\chi^{2}=0.28$ & 0.59 & $V=0.06$ \\
\hline Non-SSRI antidepressant & $8(20.0)$ & $5(16.1)$ & $\chi^{2}=0.18$ & 0.68 & $V=0.05$ \\
\hline Stimulant & $7(17.5)$ & $9(29.0)$ & $\chi^{2}=1.33$ & 0.25 & $V=0.14$ \\
\hline \multicolumn{6}{|l|}{ Current medications } \\
\hline Second-generation antipsychotic & $25(62.5)$ & $17(54.8)$ & $\chi^{2}=0.42$ & 0.51 & $V=0.08$ \\
\hline Lithium & $6(15.0)$ & $6(19.4)$ & $\chi^{2}=0.24$ & 0.63 & $V=0.06$ \\
\hline SSRI antidepressant & $3(7.5)$ & $2(6.5)$ & $\chi^{2}=0.03$ & 0.86 & $V=0.02$ \\
\hline Non-SSRI antidepressant & $1(2.5)$ & $2(6.5)$ & $\chi^{2}=0.67$ & 0.41 & $V=0.10$ \\
\hline Stimulant & $2(5.0)$ & $3(9.7)$ & $\chi^{2}=0.58$ & 0.45 & $V=0.09$ \\
\hline $\begin{array}{l}\text { NOS }=\text { not otherwise specified; } \mathrm{SSRI}=\text { select } \\
\text { Values are reported as mean } \pm \text { standard erro } \\
\text { "Kiddie Schedule for Affective Disorders and } \\
\dagger \text { Significant at } p<0.05 \text {. }\end{array}$ & $\begin{array}{l}\text { nin reuptake inhibitor. } \\
\text { enia for School-Age Chi }\end{array}$ & epression or mania ratir & & & \\
\hline
\end{tabular}

a significant cluster in the left superior parietal lobe; betweengroup differences were explained by findings of negative connectivity in adolescents with bipolar disorder and no history of cannabis use, and findings of positive connectivity in healthy controls. For the right orbitofrontal cortex seed, we found 2 significant clusters. In the right lateral occipital cortex cluster, adolescents with bipolar disorder and lifetime cannabis use displayed positive connectivity; adolescents with bipolar disorder and no history of cannabis use and healthy controls displayed negative connectivity. In the right 
Table 3: Resting-state functional connectivity, ANCOVA

\begin{tabular}{|c|c|c|c|c|c|}
\hline Seed & $\begin{array}{c}\text { MNI coordinates, } \\
x, y, z\end{array}$ & $\begin{array}{c}\text { Cluster size, } \\
\text { voxels }\end{array}$ & $p_{\mathrm{FDR}}$ & Main region & Additional region(s) \\
\hline Left amygdala & \multicolumn{5}{|c|}{ No significant clusters } \\
\hline Right amygdala & \multicolumn{5}{|c|}{ No significant clusters } \\
\hline Left nucleus accumbens & $-52,-50,36$ & 326 & $<0.001$ & Left superior parietal lobe & Left supramarginal gyrus, left angular gyrus \\
\hline Right nucleus accumbens & \multicolumn{5}{|c|}{ No significant clusters } \\
\hline Left orbitofrontal cortex & \multicolumn{5}{|c|}{ No significant clusters } \\
\hline \multirow[t]{3}{*}{ Right orbitofrontal cortex } & $36,-88,-16$ & 226 & $<0.001$ & Right lateral occipital cortex & Right occipital pole, right fusiform gyrus \\
\hline & $26,-98,0$ & 133 & 0.007 & Right occipital pole & NA \\
\hline & $-10,-104,-6$ & 77 & $0.045^{\star}$ & Left occipital pole & NA \\
\hline
\end{tabular}

ANCOVA = analysis of covariance; FDR = false discovery rate; $\mathrm{MNI}=$ Montreal Neurological Institute; NA = not applicable

${ }^{*}$ Did not survive Bonferroni multiple comparisons $(p<0.017)$.

occipital pole cluster, adolescents with bipolar disorder and lifetime cannabis use displayed positive connectivity, and adolescents with bipolar disorder and no history of cannabis use displayed negative connectivity. This study advances the literature by examining resting-state functional connectivity correlates of cannabis use in adolescents with bipolar disorder together in a comparatively large sample, and integrating a control group.

We found that adolescents with bipolar disorder and no history of cannabis use demonstrated negative connectivity relative to healthy controls, who exhibited positive connectivity between the left nucleus accumbens seed and left superior parietal lobe. The nucleus accumbens is part of the brain reward system, and it plays an important role in the processing and analysis of rewarding and reinforcing stimuli and contingencies. ${ }^{14,36,37}$ The superior parietal lobe integrates multiple sources of sensory information to form a single perception and is implicated in introspective attentional bias. ${ }^{38}$ Reward dysfunction is well established in bipolar disorder, but previous findings for resting-state functional connectivity have not robustly linked the nucleus accumbens with the parietal regions. ${ }^{39,40}$ Our finding suggests that among adolescents with bipolar disorder and no history of cannabis use, introspection may have been less rewarding than for healthy controls. Such a perspective aligns with findings of high rates of anhedonia in bipolar disorder. ${ }^{40-42}$ Negative connectivity between the nucleus accumbens and the parietal lobe, evident only in adolescents with bipolar disorder and no history of cannabis use, may explain in part why these participants have not sought out the potential rewarding effects of cannabis. However, the exact brain mechanism underlying emotion dysregulation in bipolar disorder is still not fully understood; further research is warranted to elucidate this topic. Nonetheless, with previous findings of alterations in reward network connectivity in the amygdala and frontal regions, the present findings add to the evidence of aberrant reward-related circuits in adolescent bipolar disorder. ${ }^{43-47}$

This study also found that adolescents with bipolar disorder and lifetime cannabis use exhibited positive connectivity between the right orbitofrontal cortex seed and the right lateral occipital cortex, relative to healthy controls and adolescents with bipolar disorder and no history of cannabis use, who exhibited negative connectivity. As well, adolescents with bipolar disorder and lifetime cannabis use exhibited positive connectivity relative to adolescents with bipolar disorder and no history of cannabis use, who exhibited negative connectivity between the right orbitofrontal cortex seed and the right occipital pole. The orbitofrontal cortex, part of the limbic system, is involved in executive function, particularly reward-related decision-making. ${ }^{48-51}$ The occipital lobe is primarily responsible for visual processing. ${ }^{52}$ The results from the present study could represent a stronger association between visual information and reward in adolescents with bipolar disorder and lifetime cannabis use compared to healthy controls and adolescents with bipolar disorder and no history of cannabis use. For example, adolescents with bipolar disorder and lifetime cannabis use may find visual cues of cannabis more rewarding and want to indulge in use or continue use. A previous study found elevated occipital activation during a visual attention task among young adults with chronic cannabis use relative to controls; given similar performance on visual tasks, these findings were inferred to reflect increased cognitive effort. ${ }^{53}$ The fact that this anomalous activation was normalized after prolonged abstinence from cannabis suggests that this particular finding may reflect adaptation or compensation or both, rather than predisposition to cannabis use. ${ }^{53}$ However, prospective studies and behavioural paradigms are needed to gain insight into the direction of these reward-related findings, as well as their association with other risk factors (e.g., abuse). ${ }^{54}$

\section{Limitations}

This study had several limitations that warrant consideration. Our cross-sectional, observational approach precluded us from making inferences of causation of direction. Longitudinal studies are needed to elucidate whether these connectivity patterns were a result of cannabis use or whether they precipitated cannabis use. This study did not include a control group with a history of cannabis use, limiting our ability to examine whether resting-state functional connectivity correlates of cannabis use differed between adolescents with bipolar disorder and healthy controls. We did not use urine toxicology in this study, and this could have led to underreporting of cannabis use and bias toward negative results. We 
Left nucleus accumbens seed

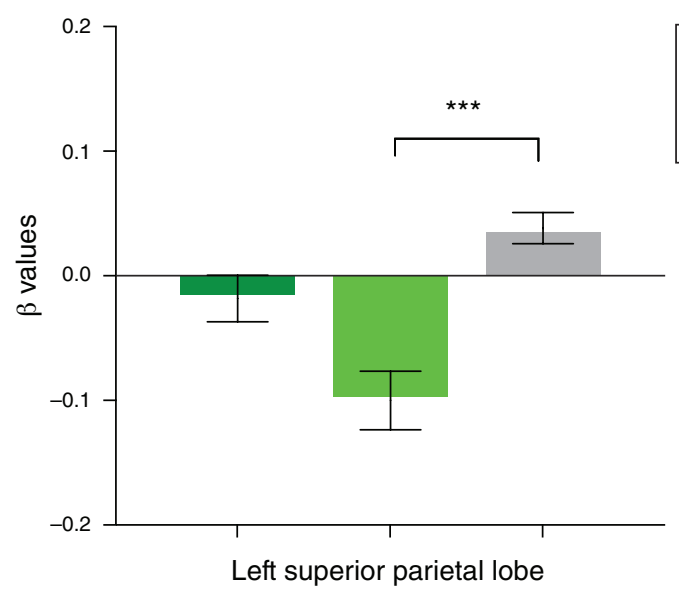

Bipolar disorder and lifetime cannabis use

Bipolar disorder and no history of cannabis use

Healthy controls

Figure 1: Significant cluster from the left nucleus accumbens seed. $\beta$ values correspond to Fisher-transformed correlation coefficient values. Error bars denote standard error of the mean. ${ }^{* * *} p<0.001$.

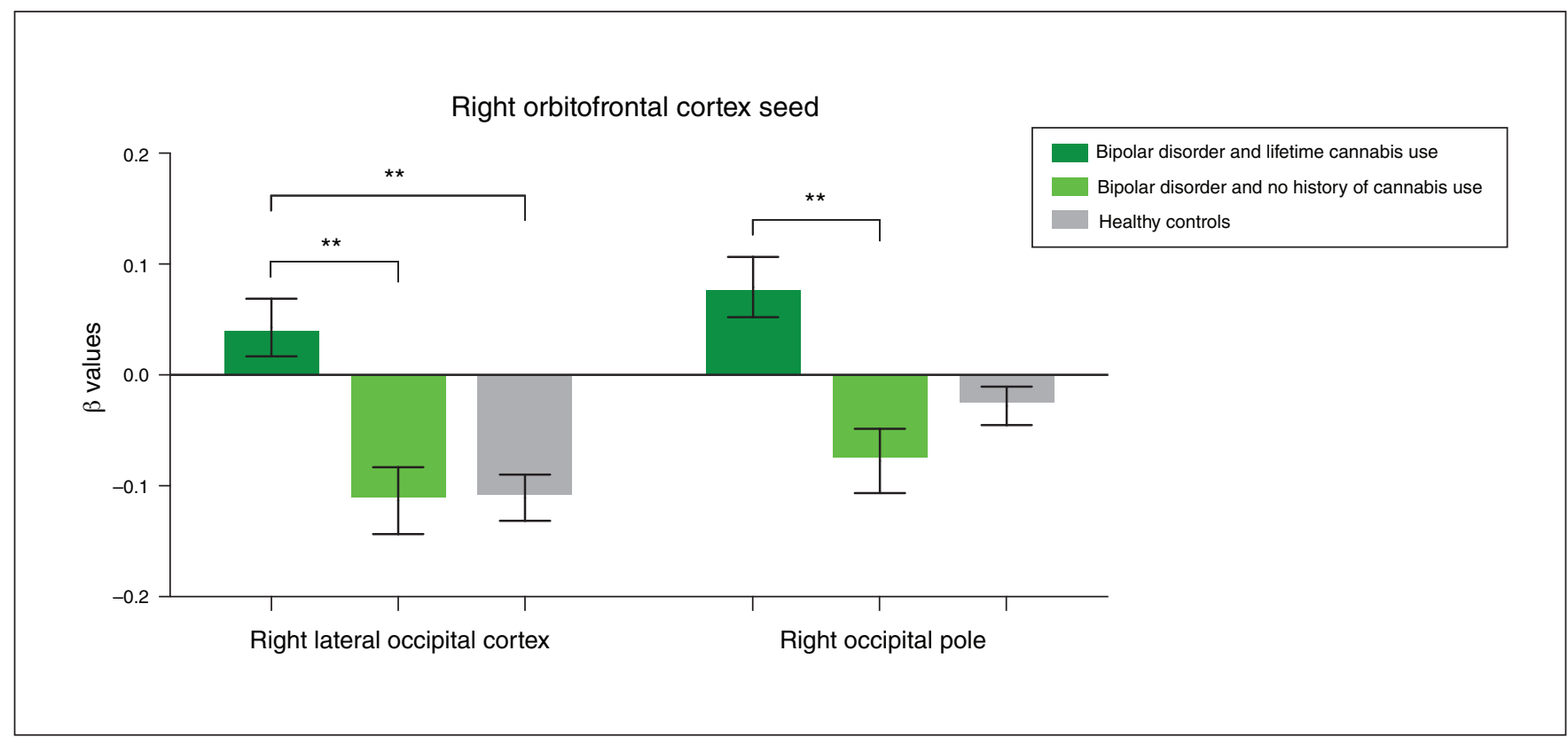

Figure 2: Significant clusters from the right orbital frontal cortex seed. $\beta$ values correspond to Fisher-transformed correlation coefficient values. Error bars denote standard error of the mean. ${ }^{* *} p<0.01$.

did not collect data about the duration, frequency or potency of cannabis use and thus could not evaluate for related associations with brain structure. The sample size of this study was not powered to conduct complicated multivariable models, which are needed to parse the independent association of cannabis use with brain structure after controlling for various clinical characteristics such as treatment, comorbidity and family psychiatry history. Relatedly, the findings for the right orbitofrontal cortex were not robust to sensitivity analyses evidence that larger, adequately powered studies are needed. Finally, other imaging modalities and approaches, such as independent component analysis or diffusion tensor imaging, are needed to provide further insight into regional connectivity and the tracts involved in cannabis use. 


\section{Conclusion}

Despite the above limitations, the present study addresses a gap in the literature by identifying resting-state functional connectivity correlates of cannabis use in adolescents with bipolar disorder. Although we were unable to make causal inferences from these results, they do provide insight into the brain basis of the clinical symptoms associated with cannabis use. Furthermore, this study provides preliminary insight into resting-state network patterns that may underlie a predisposition to or a consequence of cannabis use. Future research using prospective methodology, examining dose effects and including a group of healthy controls with a history of cannabis use, are warranted. Future research should also include a cannabis-cue task-based analysis, as well as an independent component analysis to better understand the brain connectivity related to cannabis use.

Acknowledgements: We acknowledge the contribution of the staff at the Centre for Youth Bipolar Disorder at Sunnybrook Health Sciences Centre, and we thank the adolescents and their families for their participation

Affiliations: From the Centre for Youth Bipolar Disorder, Centre for Addiction and Mental Health, (Sultan, Hird, Dimick, Goldstein); the Department of Pharmacology and Toxicology, University of Toronto, (Sultan, Dimick, Goldstein); the Faculty of Medicine, University of Toronto, (Sultan, Hird, Dimick, Goldstein); the Department of Psychiatry, University of Toronto, (Goldstein); the Department of Medical Biophysics, University of Toronto, (MacIntosh); and the Hurvitz Brain Sciences, Sunnybrook Health Sciences Centre, (MacIntosh, Goldstein) Toronto, Ont. Canada.

Competing interests: This work was supported by grants from the Ontario Mental Health Foundation, and the Canadian Institutes of Health Research awarded to Dr. B. I. Goldstein. Dr. B.I. Goldstein receives grant or research support from the Brain and Behavior Research Foundation (NARSAD), Brain Canada, the Canadian Institutes of Health Research, the Heart and Stroke Foundation, National Institute of Mental Health, and the departments of psychiatry of Sunnybrook Health Sciences Centre and the University of Toronto. Dr. Bradley MacIntosh receives grant or research support from the Canadian Institutes of Health Research, Natural Sciences and Engineering Research Council, and the Brain and Behavior Research Foundation (NARSAD). No other competing interests declared.

Contributors: A. Sultan and B. Goldstein designed the study. A. Sultan, M. Dimick, B. MacIntosh and B. Goldstein acquired the data, which all authors analyzed. A. Sultan wrote the article, which all authors reviewed. All authors approved the final version to be published and can certify that no other individuals not listed as authors have made substantial contributions to the paper.

Content licence: This is an Open Access article distributed in accordance with the terms of the Creative Commons Attribution (CC BY-NC-ND 4.0) licence, which permits use, distribution and reproduction in any medium, provided that the original publication is properly cited, the use is noncommercial (i.e., research or educational use), and no modifications or adaptations are made. See: https:/ / creativecommons.org/licenses/by-nc-nd/4.0/

\section{References}

1. Kozloff N, Cheung AH, Schaffer A, et al. Bipolar disorder among adolescents and young adults: results from an epidemiological sample. J Affect Disord 2010;125:350-4.

2. Goldstein BI, Birmaher B, Carlson GA, et al. The International Society for Bipolar Disorders Task Force report on pediatric bipolar disorder: knowledge to date and directions for future research. Bipolar Disord 2017;19:524-43.

3. Leweke FM, Koethe D. Cannabis and psychiatric disorders: it is not only addiction. Addict Biol 2008;13:264-75.

4. Goldstein BI, Bukstein OG. Comorbid substance use disorders among youth with bipolar disorder. J Clin Psychiatry 2010;71:348-58.

5. Goldstein BI, Strober MA, Birmaher B, et al. Substance use disorders among adolescents with bipolar spectrum disorders. Bipolar Disord 2008;10:469-78.

6. Wilens TE, Biederman J, Kwon A, et al. Risk of substance use disorders in adolescents with bipolar disorder. I Am Acad Child Adolesc Psychiatry 2004;43:1380-6.

7. Van Rossum I, Boomsma M, Tenback D, et al. Does cannabis use affect treatment outcome in bipolar disorder? A longitudinal analysis. J Nerv Ment Dis 2009;197:35-40.

8. Agrawal A, Nurnberger JI Jr, Lynskey MT, et al. Cannabis involvement in individuals with bipolar disorder. Psychiatry Res 2011;185:459-61.

9. Baethge C, Baldessarini RJ, Khalsa HM, et al. Substance abuse in first-episode bipolar I disorder: indications for early intervention. Am J Psychiatry 2005;162:1008-10.

10. Dickstein DP, Milham MP, Nugent AC, et al. Frontotemporal alterations in pediatric bipolar disorder: results of a voxel-based morphometry study. Arch Gen Psychiatry 2005:62:734-41.

11. Ha TH, Ha K, Kim JH, et al. Regional brain gray matter abnormalities in patients with bipolar II disorder: a comparison study with bipolar I patients and healthy controls. Neurosci Lett 2009;456:44-8.

12. Chen $\mathrm{C}-\mathrm{H}$, Suckling J, Lennox BR, et al. A quantitative meta-analysis of fMRI studies in bipolar disorder. Bipolar Disord 2011;13:1-15.

13. Mazzola-Pomietto P, Kaladjian A, Azorin JM, et al. Bilateral decrease in ventrolateral prefrontal cortex activation during motor response inhibition in mania. J Psychiatr Res 2009;43:432-41.

14. Haber SN, Knutson B. The reward circuit: linking primate anatomy and human imaging. Neuropsychopharmacology 2010;35:4-26.

15. Hartberg $\mathrm{CB}$, Lange EH, Lagerberg TV, et al. Cortical thickness, cortical surface area and subcortical volumes in schizophrenia and bipolar disorder patients with cannabis use. Eur Neuropsychopharmacol 2018;28:37-47.

16. Jarvis K, DelBello MP, Mills N, et al. Neuroanatomic comparison of bipolar adolescents with and without cannabis use disorders. J Child Adolesc Psychopharmacol 2008;18:557-63.

17. Bitter SM, Adler CM, Eliassen JC, et al. Neurofunctional changes in adolescent cannabis users with and without bipolar disorder. Addiction 2014;109:1901-9.

18. Mcneely C, Blanchard J. The teen years explained: a guide to healthy adolescent development. Baltimore (MD): Johns Hopkins Bloomberg School of Public Health; 2009. Available: www.jhsph.edu/ research/centers-and-institutes/center-for-adolescent-health/_docs/ TTYE-Guide.pdf (accessed 2020 May 12).

19. Squeglia LM, Jacobus J, Tapert SF. The influence of substance use on adolescent brain development. Clin EEG Neurosci 2009;40:31-8.

20. Crews F, He J, Hodge C. Adolescent cortical development: a critical period of vulnerability for addiction. Pharmacol Biochem Behav 2007;86:189-99.

21. Huestis MA. Cannabis (marijuana) - effects on human behavior and performance. Forensic Sci Rev 2002;14:15-60.

22. Alger BE. Getting high on the endocannabinoid system. Cerebrum 2013;14:24765232

23. Castillo PE, Younts TJ, Chávez AE, et al. Endocannabinoid signaling and synaptic function. Neuron 2012;76:70-81.

24. Wilson RI, Nicoll RA. Neuroscience: endocannabinoid signaling in the brain. Science 2002;296:678-82.

25. Engel AK, Fries P, Singer W. Dynamic predictions: oscillations and synchrony in top-down processing. Nat Rev Neurosci 2001;2:704-16.

26. Varela F, Lachaux JP, Rodriguez E, et al. The brainweb: phase synchronization and large-scale integration. Nat Rev Neurosci 2001;2:229-39.

27. Bajo R, Maestú F, Nevado A, et al. Functional connectivity in mild cognitive impairment during a memory task: implications for the disconnection hypothesis. I Alzheimer's Dis 2010;22:183-93.

28. Biswal B, Zerrin Yetkin F, Haughton VM, et al. Functional connectivity in the motor cortex of resting human brain using echoplanar MRI. Magn Reason Med 1995;34:537-41.

29. Kaufman J, Birmaher B, Brent D, et al. Schedule for Affective Disorders and Schizophrenia for School-Age Children-Present and 
Lifetime Version (K-SADS-PL): initial reliability and validity data. J Am Acad Child Adolesc Psychiatry 1997;36:980-8.

30. Axelson D, Birmaher BJ, Brent D, et al. A preliminary study of the Kiddie Schedule for Affective Disorders and Schizophrenia for School-Age Children mania rating scale for children and adolescents. J Child Adolesc Psychopharmacol 2003;13:463-70.

31. Chambers WJ, Puig-Antich J, Hirsch M, et al. The assessment of affective disorders in children and adolescents by semistructured interview. Test-retest reliability of the schedule for affective disorders and schizophrenia for school-age children, present episode version. Arch Gen Psychiatry 1985;42:696-702.

32. Shaffer D, Gould MS, Brasic J, et al. A children's global assessment scale (CGAS). Arch Gen Psychiatry 1983;40:1228-31.

33. Hollingshead A. Four factor index of social status. Yale J Sociol 2011;8:21-52.

34. Axelson D, Birmaher B, Strober M, et al. Phenomenology of children and adolescents with bipolar spectrum disorders. Arch Gen Psychiatry 2006;63:1139-48.

35. Whitfield-Gabrieli S, Nieto-Castanon A. CONN: a functional connectivity toolbox for correlated and anticorrelated brain networks. Brain Connect 2012;2:125-41.

36. Salamone JD, Correa M, Farrar A, et al. Effort-related functions of nucleus accumbens dopamine and associated forebrain circuits. Psychopharmacology (Berl) 2007;191:461-82.

37. Salamone JD, Correa M. Motivational views of reinforcement: implications for understanding the behavioral functions of nucleus accumbens dopamine. Behav Brain Res 2002;137:3-25.

38. Behrmann M, Geng JJ, Shomstein S. Parietal cortex and attention Curr Opin Neurol 2004;14:212-7.

39. Henry C, Phillips M, Leibenluft E, et al. Emotional dysfunction as a marker of bipolar disorders. Front Biosci (Elite Ed) 2012;4: 2622-30.

40. Rizvi SJ, Lambert C, Kennedy S. Presentation and neurobiology of anhedonia in mood disorders: commonalities and distinctions. Current Psychiatry Rep 2018;20:13.

41. Whitton AE, Treadway MT, Pizzagalli DA. Reward processing dysfunction in major depression, bipolar disorder and schizophrenia. Curr Opin Psychiatry 2015;28:7-12.
42. Forbes EE, Dahl RE. Altered reward function in adolescent depression: what, when and how? J Child Psychol Psychiatry 2012;53:3-15.

43. Tang Y, Ma Y, Chen X, et al. Age-specific effects of structural and functional connectivity in prefrontal-amygdala circuitry in women with bipolar disorder. BMC Psychiatry 2018;18:177.

44. Lopez-Larson MP, Shah LM, Weeks HR, et al. Abnormal functional connectivity between default and salience networks in pediatric bipolar disorder. Biol Psychiatry Cogn Neurosci Neuroimaging 2017;2:85-93.

45. Stoddard J, Hsu D, Reynolds RC, et al. Aberrant amygdala intrinsic functional connectivity distinguishes youths with bipolar disorder from those with severe mood dysregulation. Psychiatry Res 2015;231:120-5.

46. Gao W, Jiao Q, Lu S, et al. Alterations of regional homogeneity in pediatric bipolar depression: a resting-state fMRI study. BMC Psychiatry 2014;14:222.

47. Xiao Q, Zhong Y, Lu D, et al. Altered regional homogeneity in pediatric bipolar disorder during manic state: a resting-state fMRI study. PLoS One 2013;8:e57978.

48. Rolls ET. The orbitofrontal cortex and reward. Cerebral Cortex 2000;3:284-94.

49. Rolls ET. The functions of the orbitofrontal cortex. Brain Cogn 2004;55:11-29.

50. Kennerley SW, Walton ME. Decision making and reward in frontal cortex: complementary evidence from neurophysiological and neuropsychological studies. Behav Neurosci 2011;125:297-317.

51. Rolls ET, Grabenhorst F. The orbitofrontal cortex and beyond: from affect to decision-making. Prog Neurobiol 2008;86:216-44.

52. Rehman A, Al Khalili Y. Neuroanatomy, occipital lobe. Treasure Island (FL); StatPearls Publishing: 2019. Available: www.ncbi.nlm. nih.gov/pubmed/31335040 (accessed 2020 Jun. 5).

53. Chang L, Yakupov R, Cloak C, et al. Marijuana use is associated with a reorganized visual-attention network and cerebellar hypoactivation. Brain 2006;129:1096-112.

54. Kirsch DE, Tretyak V, Radpour S, et al. Childhood maltreatment, prefrontal-paralimbic gray matter volume, and substance use in young adults and interactions with risk for bipolar disorder. Sci Rep 2021;11:123. 